\title{
Separate Effects of the Microkeratome Incision and Laser Ablation on the Eye's Wave Aberration
}

\author{
JASON PORTER, MS, SCOTT MACRAE, MD, GEUNYOUNG YOON, PHD, \\ CYNTHIA ROBERTS, PHD, IAN G. COX, PHD, AND DAVID R. WILLIAMS, PHD
}

- PURPOSE: To study the optical changes induced by the microkeratome cut, the subsequent laser ablation, and the biomechanical healing response of the cornea in normal laser in situ keratomileusis (LASIK) eyes.

- DESIGN: Prospective randomized clinical trial.

- METHODS: A Hansatome microkeratome was used to cut a corneal flap in one eye (study eye) of 17 normal myopic patients and a subsequent laser ablation was performed 2 months after this initial microkeratome incision. Control eyes received conventional LASIK treatments at the latter time point. The wave aberration of both the study and contralateral control eyes were measured over a $6-\mathrm{mm}$ pupil with a Shack-Hartmann wavefront sensor for all preoperative, postflap cut, and postablation visits.

- RESULTS: The eye's higher order aberrations had a small, but significant increase $(P=.03)$ of approximately $30 \% 2$ months after cutting a flap. No systematic changes were observed in nearly all Zernike coefficients from their preoperative levels at 2 months postflap cut. A significant difference between the study and control eyes was observed for one trefoil mode, $Z_{3}^{3}(P=.04)$.

- CONCLUSIONS: There was a wide variation in the response of individual Zernike modes across patients after cutting a flap. The majority of spherical aberration induced by the LASIK procedure seems to be due to the laser ablation and not the microkeratome cut. In addi-

Accepted for publication Feb 12, 2003.

InternetAdvance publication at ajo.com Feb 19, 2003.

From The Institute of Optics (J.P.), Department of Ophthalmology (S.M., G.Y.), and Center for Visual Science (D.R.W.), University of Rochester, Rochester, New York; Department of Ophthalmology (C.R.), Ohio State University, Columbus, Ohio; and Bausch \& Lomb (I.G.C.), Rochester, New York.

Supported by the National Institutes of Health grants EY01319 and EY07125, and a research grant from Bausch \& Lomb. Jason Porter, Geunyoung Yoon, Cynthia Roberts, David Williams, and Scott MacRae have served as consultants for Bausch \& Lomb. In addition, the University of Rochester has a licensing agreement with Bausch \& Lomb for the customized correction of higher order aberrations.

Inquiries to Scott MacRae, MD, StrongVision, 100 Meridian Centre, Suite 125, Rochester, NY 14618; fax: (585) 756-1975; e-mail: scott_ macrae@urmc.rochester.edu tion, the total and higher order root mean square of wavefront errors were nearly identical for both the study and control eyes 3-months after the laser ablation, indicating that a procedure in which the incision and the ablation are separated in time to better control aberrations does not compromise the outcome of a conventional LASIK treatment. (Am J Ophthalmol 2003;136: 327-337. () 2003 by Elsevier Inc. All rights reserved.)

E VEN THOUGH EARLY CUSTOMIZED ABLATION RESULTS have been encouraging, further refinements of wavefront-guided laser in situ keratomileusis (LASIK) procedures are needed to obtain an aberration-free ablative correction. ${ }^{1-4}$ It has been well established that conventional photorefractive keratectomy (PRK) and LASIK procedures induce higher order aberrations, including increased amounts of spherical aberration, despite their accurate correction of spherocylindrical errors..$^{5-10}$ To date, little evidence has been presented to determine the origins of these induced higher order aberrations. Consequently, not much is known about the importance or relative contribution of the two separate effects of creating a microkeratome flap and performing a subsequent laser ablation on the overall optical quality of an eye receiving a LASIK treatment. Developing a firm understanding of the aberrations induced by these two processes is crucial for achieving an optimal, customized correction of the eye's higher order aberrations with laser refractive surgery.

Studies have been conducted to investigate structural changes in corneal shape due to the laser ablation in refractive surgery procedures. Howland and coworkers ${ }^{11}$ found that changes made in the midperipheral and peripheral cornea could create biomechanical changes in the central cornea in radial keratotomy. In PRK and LASIK procedures, central corneal shape can be altered by shape changes occurring outside of the ablation zone. The laser ablation itself creates a thickening of the peripheral stroma (that is, an increase in corneal elevation outside of the ablation zone) and a secondary steepening of the midperipheral cornea. This peripheral steepening results in a 
flattening of the central cornea and consequently a shift toward hyperopia. ${ }^{12,13}$ These peripheral and central shape changes, along with the unintended hyperopic shift, can occur even for a PTK type ablation, which is not designed to change corneal curvature. ${ }^{14-17}$ As a result, the biomechanical response of the cornea to the laser ablation will yield central flattening and hyperopic shifts for both myopic and hyperopic procedures. Few reports have discussed the effects of these biomechanical changes on aberrations other than defocus, or sphere.

We sought to determine how the eye's aberrations changed as a result of the microkeratome flap-cut and the laser ablation. The first experimental stage provided information on the degree of aberration change resulting only from the corneal flap-cut and the subsequent readhesion of the flap to the cornea. The second stage offered further information on the amount of aberrations that are induced in LASIK solely due to the laser ablation. Comparing postablation aberration data from the study eyes with data from the normal, one-step LASIK-treated, contralateral control eyes might provide preliminary insight for whether conventional LASIK yields better optical performance when performed as a one- or two-step procedure. Finally, this study could also provide insight as to whether the majority of spherical aberration induced after a LASIK procedure is a result of the microkeratome incision or the laser ablation.

\section{METHODS}

SEVENTEEN HEALTHY MYOPIC PATIENTS BETWEEN 30 AND 50 years of age were enrolled in an institutional review board-monitored study. A full informed consent was obtained for each patient. The myopia ranged between -0.75 and -6.75 diopters (D) with less than $-2.50 \mathrm{D}$ of astigmatism.

The study was divided into two stages: a postmicrokeratome incision (or postflap cut) stage and a postlaser ablation stage. In the first stage, patients received a microkeratome incision with a superior hinge in only one eye (study eye) while the contralateral eye (control eye) was left untreated. A Hansatome Microkeratome (Bausch \& Lomb, Rochester, New York, USA) was used to perform the cut. The study eye for each patient was chosen at random. All study eyes received a $180-\mu \mathrm{m}$ microkeratome plate depth incision with a flap diameter of $9.5 \mathrm{~mm}$ or $8.5 \mathrm{~mm}$, and no subsequent laser treatment was performed after the incision.

The second stage of the study was conducted at least 2 months after the initial microkeratome incision was made in the study eye. In the second stage, the microkeratome flap was lifted in the study eyes and a conventional myopic excimer ablation (Technolas 217c; Bausch \& Lomb) was performed based on the preoperative and 2-month postmicrokeratome incision manifest refractions. At that time,
TABLE 1. Average Preoperative Manifest Refractions and Attempted Corrections for Study and Control Eyes

\begin{tabular}{llllll}
\hline & \multicolumn{2}{c}{$\begin{array}{c}\text { Preoperative Manifest } \\
\text { Refraction }\end{array}$} & & \multicolumn{2}{c}{$\begin{array}{c}\text { Attempted Laser } \\
\text { Correction }\end{array}$} \\
\cline { 2 - 3 } \cline { 5 - 6 } Eyes & Sphere & Cylinder & & Sphere & Cylinder \\
\hline Study & $-2.62 \mathrm{D}$ & $-0.50 \mathrm{D}$ & & $-2.55 \mathrm{D}$ & $-0.67 \mathrm{D}$ \\
Control & $-2.66 \mathrm{D}$ & $-0.65 \mathrm{D}$ & & $-2.61 \mathrm{D}$ & $-0.70 \mathrm{D}$ \\
\hline \multicolumn{2}{l}{$\mathrm{D}=$ diopters. } & & & & \\
\hline
\end{tabular}

the contralateral control eye was treated with a conventional LASIK treatment based on the most recent manifest refraction. The average preoperative manifest refractions and attempted corrections for both the study and control eyes are presented in Table 1 .

Manifest refractions and uncorrected and best-corrected visual acuities were performed preoperatively and at each postoperative examination. A Shack-Hartmann wavefront sensor (Zywave; Bausch \& Lomb) was used to measure the monochromatic wave aberration of both the study and control eyes under scotopic conditions with dilation (2.5\% neosynephrine). The wave aberration was expressed as a Zernike polynomial up to and including fifth order aberrations (18 Zernike terms) over a 6-mm pupil. The Zernike polynomials were expressed according to the standard normalized polynomials and representation established by the VSIA Standards Taskforce team. ${ }^{18}$ Wavefront analysis was made comparable for right and left eyes by creating the mirror image of the wavefronts from all left eyes (that is, flipping the signs of all appropriate Zernike coefficients that exhibit odd symmetry about the vertical axis) and performing the analysis. All measurements were made in the study and the contralateral control eyes preoperatively (Pre), at 20 minutes (20 min), 1 day (1D), 1 week (1W), 1 month (1M), and 2 months (2M) postmicrokeratome incision. The same measurements were also performed 1 day (1DLSK), 1 week (1WLSK), 1 month (1 MLSK), and 3 months (3 MLSK) postlaser ablation.

- STATISTICAL ANALYSIS: To determine which aberrations significantly changed due to the flap cut, two-tailed $t$ tests were used to test for significance when comparing postoperative differences in aberrations (relative to preop) between the study and control eyes 2 months postflap cut. The same tests were used to investigate potential significant changes between the study and control eyes 3 months after the laser ablation. Finally, paired, two-tailed $t$ tests were used to determine significant changes in aberrations from preoperative levels within the study eyes and the control eyes 3 months postablation. Values of $P<.05$ were considered to be significant. 


\section{Total Aberrations}

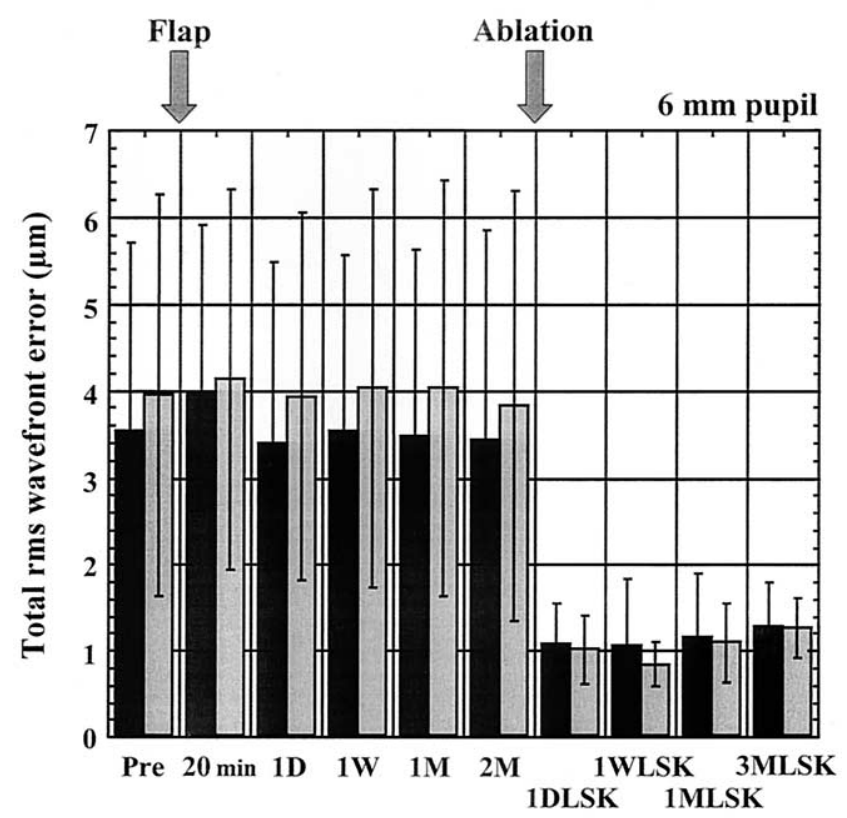

Time

FIGURE 1. Average total root mean square (rms) of the wavefront error for both study eyes ( $\square$ ) and control eyes ( $\square$ ) for a 6 -mm pupil. Error bars represent \pm 1 standard deviation from the mean. Time points were preoperative (Pre), 20 minutes (20 min), 1 day (1D), 1 week (1W), 1 month (1M), and 2 months (2M) postmicrokeratome incision; and 1 day (1DLSK), 1 week (1WLSK), 1 month (1 MLSK), and 3 months ( $3 \mathrm{MLSK}$ ) postlaser ablation. There was no significant difference between the total $\mathrm{rms}$ wavefront error in the study and control eyes 2 months $(2 \mathrm{M})$ postflap cut $(P=.95)$.

\section{RESULTS}

- TOTAl ABERRATIONS: The evolution of the total rms wavefront error in the study and control eyes for the postflap cut and postablation stages is illustrated in Figure 1. No significant change was observed 2 months postflap cut between the study and control eyes relative to their preoperative levels. The average preoperative total $\mathrm{rms}$ wavefront error ( \pm 1 standard deviation) for the study eyes was $3.55 \pm 2.159 \mu \mathrm{m}$, whereas that for the contralateral control eyes was $3.96 \pm 2.309 \mu \mathrm{m}$. Two months after the flap cut, the average total root mean square (rms) of the wavefront error fell, but not significantly $(P=.95)$, in the study and control groups to $3.44 \pm 2.421 \mu \mathrm{m}$ and $3.83 \pm$ $2.481 \mu \mathrm{m}$, respectively. After relifting the flap and performing a conventional ablation, the total $\mathrm{rms}$ wavefront error in the study eyes was reduced to $1.30 \pm 0.498 \mu \mathrm{m}$ (55\% average reduction) 3 months postablation. The contralateral control eyes had a similar reduction to 1.27 $\pm 0.350 \mu \mathrm{m}$ (60\% average reduction) 3 months after performing a conventional LASIK procedure. The ob-
Manifest Spherical Refraction

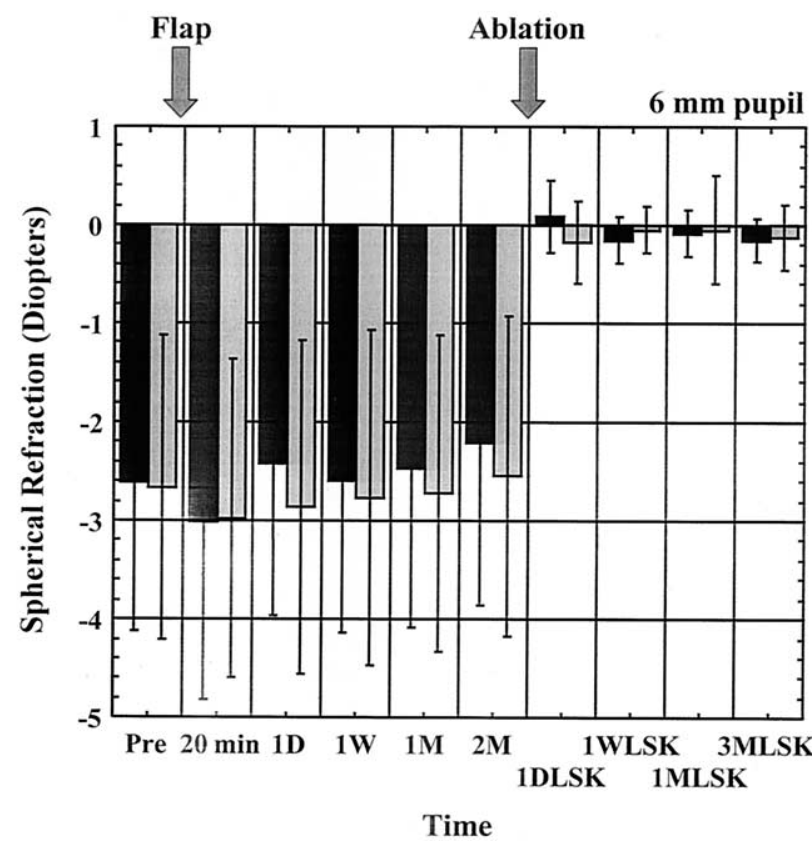

FIGURE 2. Mean manifest spherical refraction for both study eyes $(\square)$ and control eyes $(\square)$ for a 6-mm pupil. Error bars represent \pm 1 standard deviation from the mean. Time points were preoperative (Pre), 20 minutes $(20 \mathrm{~min}), 1$ day (1D), 1 week ( $1 \mathrm{~W}), 1$ month $(1 \mathrm{M})$, and 2 months $(2 \mathrm{M})$ postmicrokeratome incision; and 1 day (1DLSK), 1 week (1WLSK), 1 month (1 MLSK), and 3 months ( 3 MLSK) postlaser ablation. Two months $(2 \mathrm{M})$ postflap cut, the study eyes experienced a significant hyperopic shift (relative to preoperative) of 0.28 diopters $(P=.01)$ compared with the controls. However, both groups were not statistically different from each other 3 months (3 MLSK) after the ablation.

served decreases after the laser ablation were primarily due to the reduction in each patient's sphere and cylinder (defocus and astigmatism), as these aberrations are the largest contributors to the eye's total rms wavefront error before surgery. ${ }^{19,20}$

- LOWER ORDER ABERRATIONS-SPHERE AND CYLIN DER: No significant differences (relative to preoperative levels) were observed for the Zernike defocus or astigmatism modes at the 2-month postflap cut time point between the study and control eyes. Figure 2 demonstrates the evolution of the average manifest spherical refraction for both groups. Two months after the flap cut there was a significant hyperopic shift of $0.28 \pm 0.36 \mathrm{D}(P=.01)$ in the manifest spherical refraction of the study eyes when compared with the control eyes. Two patients, however, opted not to have laser treatment in their study eyes due to a $0.50-\mathrm{D}$ reduction in myopia that occurred 2 months after the initial flap cut. In addition, there was no significant difference between the study and control groups in mani- 


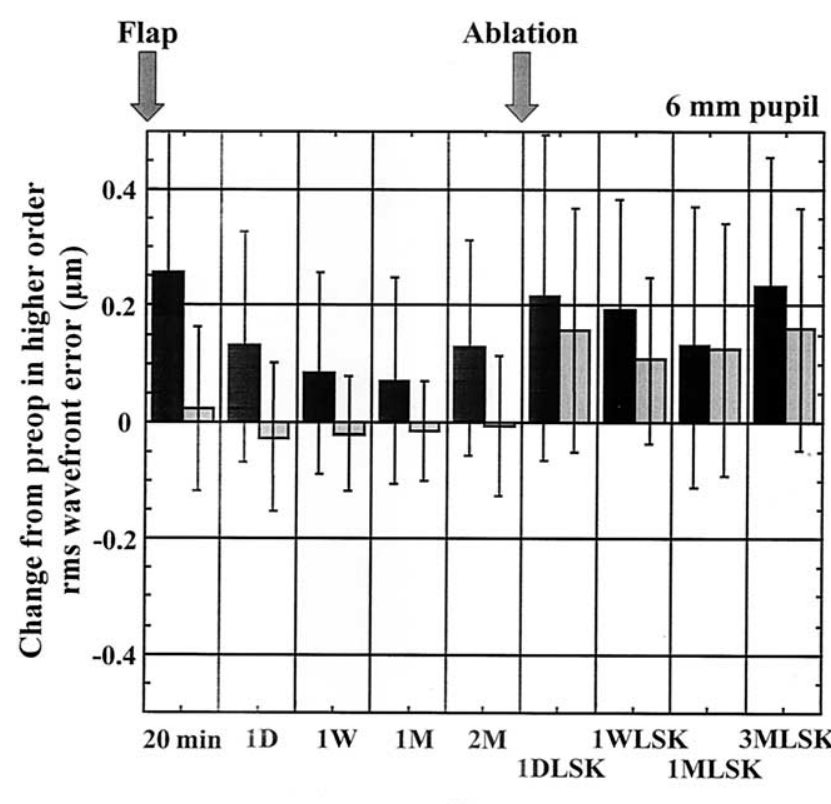

Time

FIGURE 3. Average change in higher order root mean square (rms) of the wavefront error from preoperative levels for both study eyes ( $\square$ ) and control eyes $(\square$ ) for a 6-mm pupil. Positive and negative values indicate an average increase or decrease, respectively, in higher order rms wavefront error from preoperative values. Error bars represent \pm 1 standard deviation from the mean. Time points were preoperative (Pre), 20 minutes (20 min), 1 day (1D), 1 week (1W), 1 month (1M), and 2 months (2M) postmicrokeratome incision; and 1 day (1DLSK), 1 week (1WLSK), 1 month (1 MLSK), and 3 months ( $3 \mathrm{MLSK}$ ) postlaser ablation. The study eyes demonstrated a small but significant increase $(P=.01)$ in higher order aberrations when compared with the control eyes 2 months (2M) after the microkeratome incision. No significant difference was noted between these two groups 3-months postablation (3 MLSK), even though both groups had significantly increased higher order aberrations relative to preoperative levels.

fest spherical refraction (average values of $-0.15 \pm 0.22 \mathrm{D}$ and $-0.13 \pm 0.33 \mathrm{D}$, respectively) 3 months postlaser ablation.

- HIGHER ORDER ABERRATIONS: The average change in higher order rms wavefront error (relative to preoperative levels) across eyes for both groups is illustrated in Figure 3. Positive and negative values indicate an average increase or decrease, respectively, in higher order rms wavefront error from preoperative, whereas a value of zero would indicate no average change from preoperative levels. The study eyes had a small but significant increase $(P$ $=.03$ ) in higher order rms wavefront error of $0.128 \pm$ $0.1847 \mu \mathrm{m}$ relative to the contralateral control eyes 2 months postflap cut. This represented an average increase of approximately $30 \%$ across study eyes compared with their average amount of preoperative higher order $\mathrm{rms}$ $(0.438 \pm 0.1924 \mu \mathrm{m})$.

Both groups exhibited significant increases in higher order rms wavefront error 3 months postablation when compared with their average preoperative values. There was a significant average increase $(P=.002)$ across the study eyes of $0.233 \pm 0.2239 \mu \mathrm{m}$ at the 3 -month postablation time point. The control group also had a significant increase at 3 months postablation $(P=.01)$ in higher order aberrations, relative to their preoperative values, of $0.160 \pm 0.2076 \mu \mathrm{m}$. The differences in higher order rms wavefront error between the study and control groups were not significant when compared with all four postablation time points (that is, 1 day $[P=.55], 1$ week $[P=.18], 1$ month $[P=.95]$, and 3 months $[P=.39]$ postablation).

Figure $4 \mathrm{~A}$ reveals the average change in all higher order aberrations (third through fifth order), third order, fourth order and fifth order and the rms of spherical aberration for only the study eyes at three postoperative time points: 20 minutes after the flap cut, 2 months postmicrokeratome incision, and 3 months postlaser ablation. The same information is plotted at identical time periods in Figure 4B for the control eyes. The study eyes displayed a significant increase, relative to the unoperated, control eyes, in higher-order $(P=.007)$, third order $(P=.02)$, fourth order $(P=.03)$, and fifth order $(P=.003) \mathrm{rms}$ wavefront error 20 minutes after the microkeratome incision was made, although spherical aberration did not significantly change $(P=.30)$. The majority of the change in higher order aberrations was dominated by the third order aberrations. Two months after the flap cut, only the higher order and third order aberrations remained significantly increased with respect to the controls $(P=.03$ for both cases).

Three months postablation, there was another significant increase in the rms wavefront error for spherical aberration, higher order, third, fourth, and fifth order aberrations when compared with preoperative levels in the study eyes. The control eyes demonstrated a similar increase in spherical aberration, higher order, fourth and fifth order rms wavefront error when comparing post-LASIK to preoperative values (Figure 4B). The increase in third order rms wavefront error in the control eyes $(0.084 \pm$ $0.1927 \mu \mathrm{m})$ was neither significant nor as large as in the study eyes $(0.200 \pm 0.2356 \mu \mathrm{m})$. However, as with the higher-order aberrations, the differences in the third order rms wavefront error between the study and control eyes were not significant when comparing both groups at the final 3-month postlaser treatment visit.

In addition to examining changes in the overall amounts of higher order aberrations due to the flap cut and laser ablation, we also investigated changes for each measured Zernike mode. No systematic changes were observed in nearly all Zernike terms across patients after cutting a corneal flap. There were no significant differences in the change of either horizontal or vertical coma $\left(Z_{3}^{1}\right.$ and 


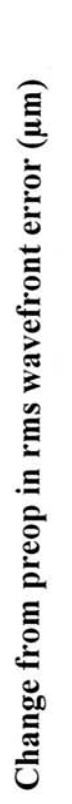

A

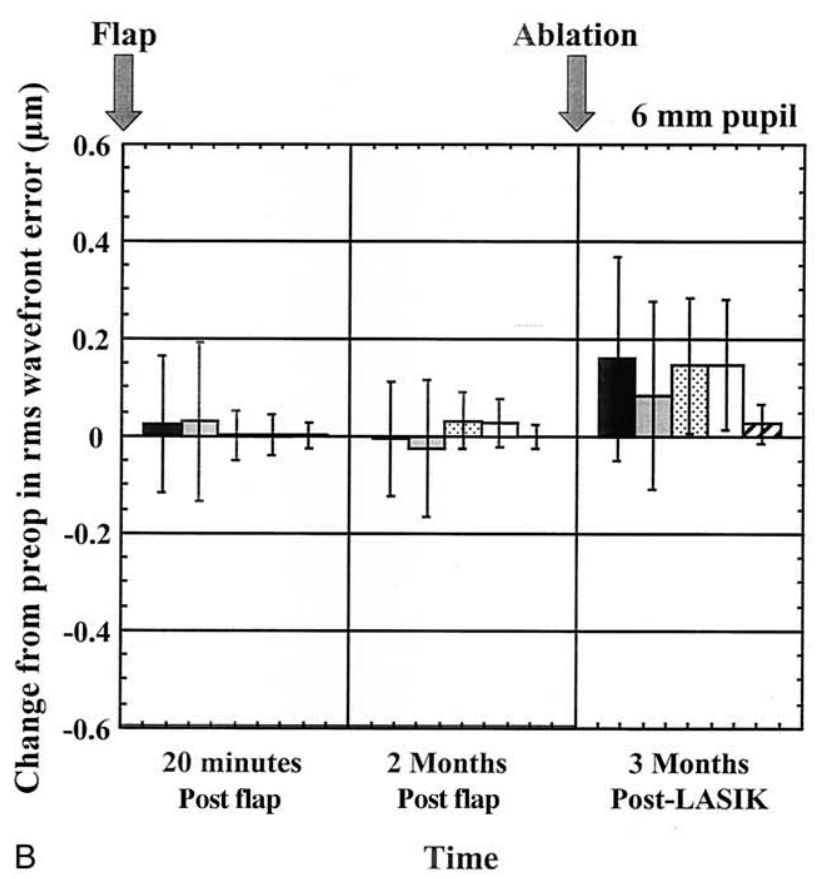

$\mathrm{Z}_{3}^{-1}$ ) between the study and control eyes 2 months after cutting the flap. The average changes (from preoperative) in vertical coma, $Z_{3}^{-1}$, for both the study and control groups are illustrated in Figure 5. Although the study eyes experienced a significantly sharp increase in vertical coma 20 minutes after the flap cut, this effect subsided in 24 hours. Two months after the microkeratome incision, the changes in vertical coma observed in the study and control eyes were almost identical. In addition, both groups had nearly the same changes in vertical coma 3 months postlaser ablation, with the induced changes being signif-
FIGURE 4. Average change in (A) study eyes and (B) control eyes for higher order root mean square (rms) of wavefront error $(\square)$, third order rms wavefront error $(\square)$, rms error of spherical aberration (dotted bars), fourth order rms wavefront error $(\square)$, and fifth order rms wavefront error $(\approx)$ at 20-minutes and 2 -months postflap cut, and 3-months postablation $(6-\mathrm{mm}$ pupil). Error bars represent \pm 1 standard deviation from the mean. All categories in the study eye, except for spherical aberration, were significantly differently from the control eyes 20-minutes postmicrokeratome incision, but only the higher order and third order rms wavefront errors remained significantly different at the 2-month postflap time point. Three months after the ablation no categories in the study eyes were significantly different from the control eyes. Nearly all categories were significantly different from preoperative levels for both groups at this postablation time point.

icantly different from their preoperative values for the study eyes $(P=.02)$ and nearly significantly different for the controls $(P=.05)$.

The only Zernike mode that displayed a systematic change after the flap-cut was trefoil, $Z_{3}^{3}$. The average changes (from preoperative) in trefoil for the study and control eyes are illustrated in Figure 6. As with vertical coma, the study eyes had a significant change in trefoil immediately after the flap was made (20 minutes), with this change relaxing back toward preoperative levels within the first 24 hours. This shift in trefoil did not completely return to preoperative levels, however. The study eyes had a significant negative shift in trefoil relative to the control eyes $(P=.04)$ of $-0.106 \pm 0.1679 \mu \mathrm{m}$, on average, 2 months after the flap cut. A majority of the study eyes also experienced an increase in the magnitude of trefoil. There were no significant differences in the change in trefoil (relative to preop) between the study and control groups after the laser ablation.

Finally, the microkeratome incision did not induce significant amounts of spherical aberration in the study group. Figure 7 illustrates the changes in spherical aberration, $Z_{4}^{0}$, relative to preoperative levels, for the study and control eyes. The average preoperative amount of spherical aberration was $0.218 \pm 0.1424 \mu \mathrm{m}$ for the study eyes and $0.218 \pm 0.1359 \mu \mathrm{m}$ for the controls. Two months after the initial flap cut, the change in spherical aberration from preoperative for the study group was not significantly different from the contralateral control group $(P=.36)$. No significant difference was observed when comparing the changes in spherical aberration between the study and control eyes at the 3-month postablation time point. More importantly, both groups experienced significant increases in spherical aberration from their original preoperative values 3 months after the ablation $(P=.0004$ and $P=$ .0003 for study and control eyes, respectively). This result suggests the majority of the increase in spherical aberration post-LASIK is due to the actual laser ablation and not the microkeratome incision. 


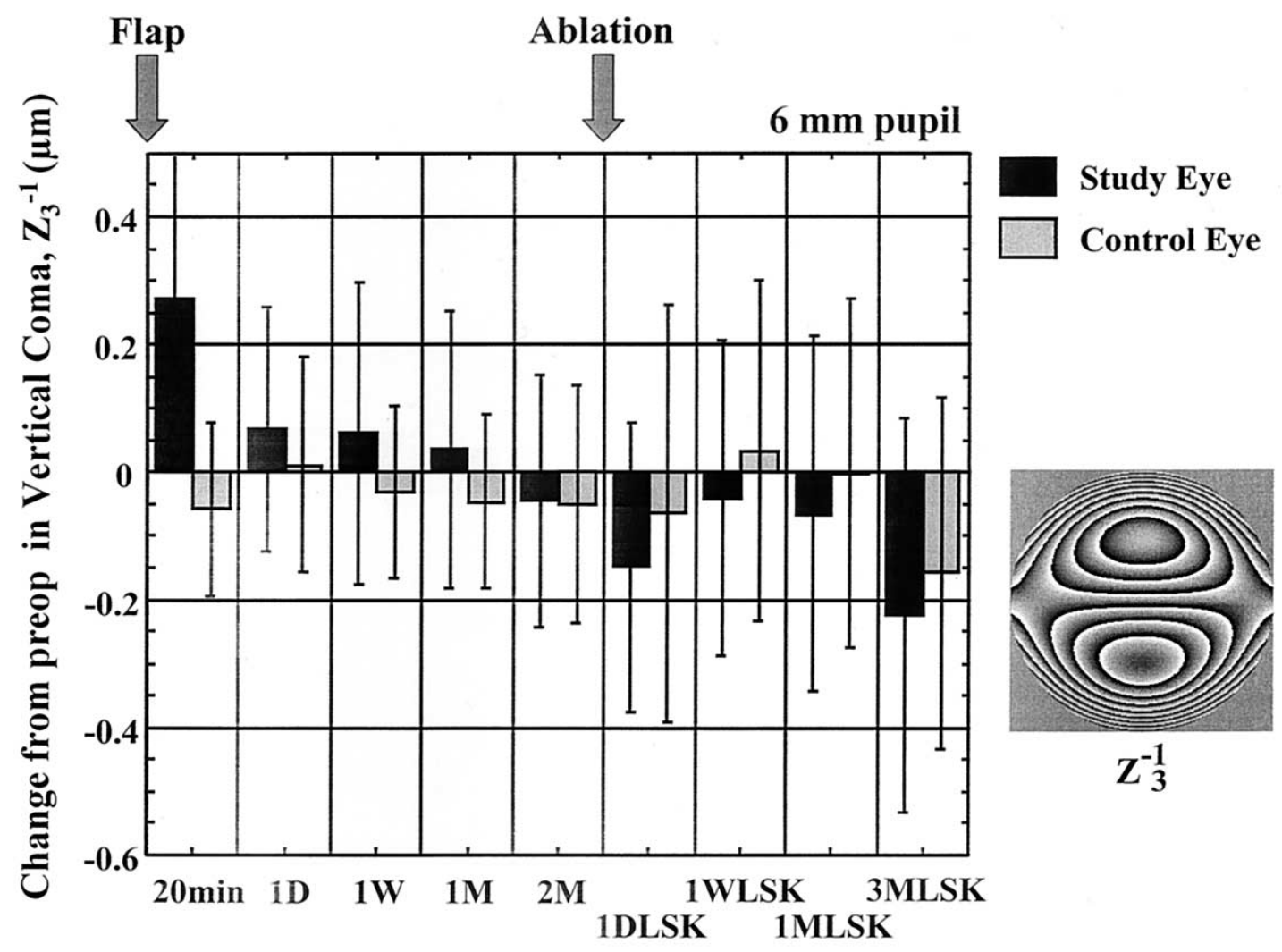

Time

FIGURE 5. Average change in vertical coma, $Z_{3}^{-1}$, from preoperative levels for both study eyes ( $\square$ ) and control eyes ( $\square$ ) for a 6-mm pupil. Positive and negative values indicate an average positive or negative shift, respectively, in vertical coma relative to preoperative values. Error bars represent \pm 1 standard deviation from the mean. Time points were 20 minutes (20 min), 1 day (1D), 1 week $(1 \mathrm{~W}), 1$ month (1M), and 2 months (2M) postmicrokeratome incision; and 1 day (1DLSK), 1 week (1WLSK), 1 month (1 MLSK), and 3 months ( 3 MLSK) postlaser ablation. There was a sharp increase in vertical coma 20-minutes after the flap was created in the study eye group. This increase quickly subsided the next day and had an average value equivalent to the control eye group 2-months after the microkeratome cut. Three-months after the ablation there was no significant difference in vertical coma between the study and control groups.

\section{DISCUSSION}

THIS STUDY NOTED THAT THERE WAS A LARGE REDUCTION in the total rms wavefront error after the excimer laser ablation both in the study eyes, treated initially with a microkeratome incision followed by an ablation, and the contralateral control eyes, treated with conventional LASIK (Figure 1). This reduction was expected given that sphere and cylinder constitute nearly $90 \%$ of the total variance of the wave aberration in the population of normal eyes, with higher order aberrations comprising the remaining $10 \% .{ }^{19}$ Our cohort of patients closely matched these results, as the preoperative second order aberrations (sphere and cylinder) constituted, on average, approximately $85 \%$ of the total rms wavefront error. Three months after the laser surgery, the higher order aberrations made up almost $50 \%$ of the total rms wavefront error, on average across patients, while the second order aberrations made up the remaining 50\%. This change in aberration structure was primarily caused by the reduction in sphere and cylinder and a moderate average increase in higher order aberrations as a result of the treatment. These post-LASIK trends are consistent with previously mentioned studies documenting that conventional PRK and LASIK procedures cause an increase in higher order aberrations. $^{5-10}$

Total rms wavefront error changed negligibly with the creation of the microkeratome flap, however, if the average preoperative total rms value of $3.55 \pm 2.159 \mu \mathrm{m}$ is compared with the average 2 -month postoperative flap cut value of $3.44 \pm 2.421 \mu \mathrm{m}$ in the study group (Figure 1). Clinically, patients confirmed this observation by noting that they were able to see well with the same spectacle correction they had used preoperatively and that their manifest refractions postflap cut were similar. Two patients elected not to receive the second stage treatment of relifting the flap and undergoing an ablation in their study eyes. Both of these eyes were low myopes preoperatively, 


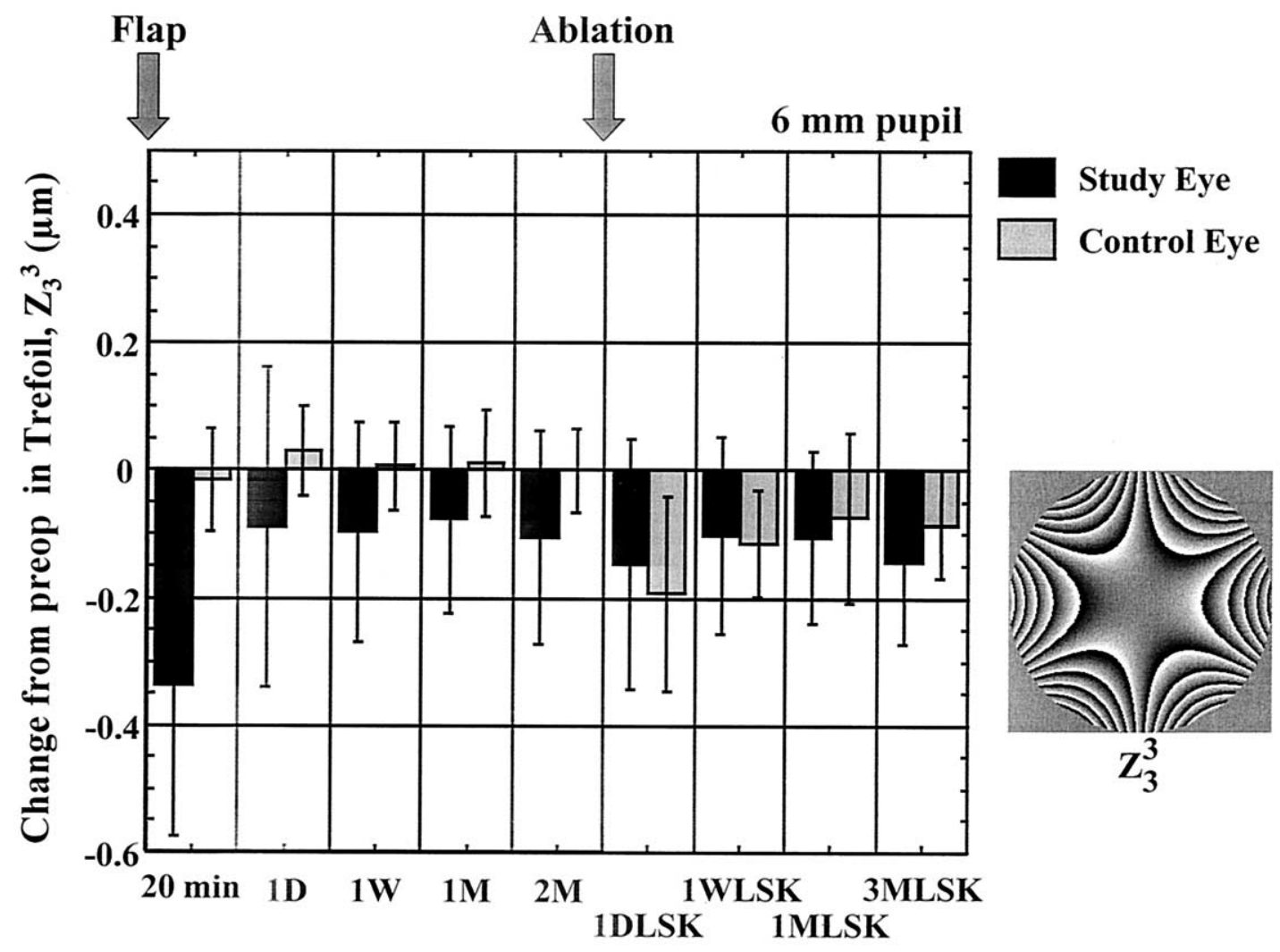

Time

FIGURE 6. Average change in trefoil, $Z_{3}^{3}$, from preoperative levels for both study eyes ( $\square$ ) and control eyes ( $\square$ ) for a 6-mm pupil. Positive and negative values indicate an average positive or negative shift, respectively, in trefoil relative to preoperative values. Error bars represent \pm 1 standard deviation from the mean. Time points were 20 minutes (20 min), $1 \mathrm{day}(1 \mathrm{D}), 1 \mathrm{week}(1 \mathrm{~W}), 1$ month (1M), and 2 months (2M) postmicrokeratome incision; and 1 day (1DLSK), 1 week (1WLSK), 1 month (1 MLSK), and 3 months ( 3 MLSK) postlaser ablation. The study eyes demonstrated a small but significant negative shift $(P=.03)$ in this trefoil mode when compared with the control eyes 2 months (2M) after the microkeratome incision, as most study eyes experienced an increase in the magnitude of trefoil. No significant difference was noted between these two groups 3-months postablation (3 MLSK).

and both experienced subjective improvements in visual acuity 2 months postmicrokeratome incision. The first had a manifest refraction of $-1.00-0.25 \times 90$ degrees $D$ that was reduced to $-0.50 \mathrm{D}$ sphere 2 months after the microkeratome incision. The second eye was $-1.25-0.50 \times 30$ degrees $\mathrm{D}$ preoperatively and experienced a myopic reduction of 0.50 diopters. These two patients received conventional LASIK treatments in the contralateral control eyes with manifest refractions of plano 3 months after LASIK.

The experimental design and results also provide insight as to whether conventional LASIK procedures yield better postoperative outcomes if performed as a one- or two-stage procedure. For our cohort of patients, no significant differences were observed in the total or higher order rms wavefront error or in any individual Zernike mode 3 months after the ablation when comparing the study group (two-stage procedure) to the controls (one-stage procedure). These results suggest that the postablation aberration outcomes in a conventional LASIK procedure are independent of whether the treatment is performed in one or two stages.

The higher order aberrations increased sharply immediately after the flap was created ( 20 minutes) by an average rms wavefront error of $0.258 \pm 0.2503 \mu \mathrm{m}$ (Figure 3). The higher order rms wavefront error in the study eyes remained significantly elevated compared with the control eyes 2 months after the microkeratome incision. This persistent increase in higher order aberrations was caused mostly by increases in third order aberrations, as illustrated in Figure 4A. The same sharp increase in higher order aberrations was also noted 1 day after the ablation (Figure $3,1 \mathrm{DLSK})$ in the study eyes as well as in the LASIKtreated control eyes. This sudden increase in higher order aberrations may account for some of the blurred vision noted subjectively in the early postoperative period and seems to subside with time (Figure 3).

There was also a significant increase in vertical coma, $\mathrm{Z}_{3}^{-1}, 20$-minutes after the microkeratome incision (20 


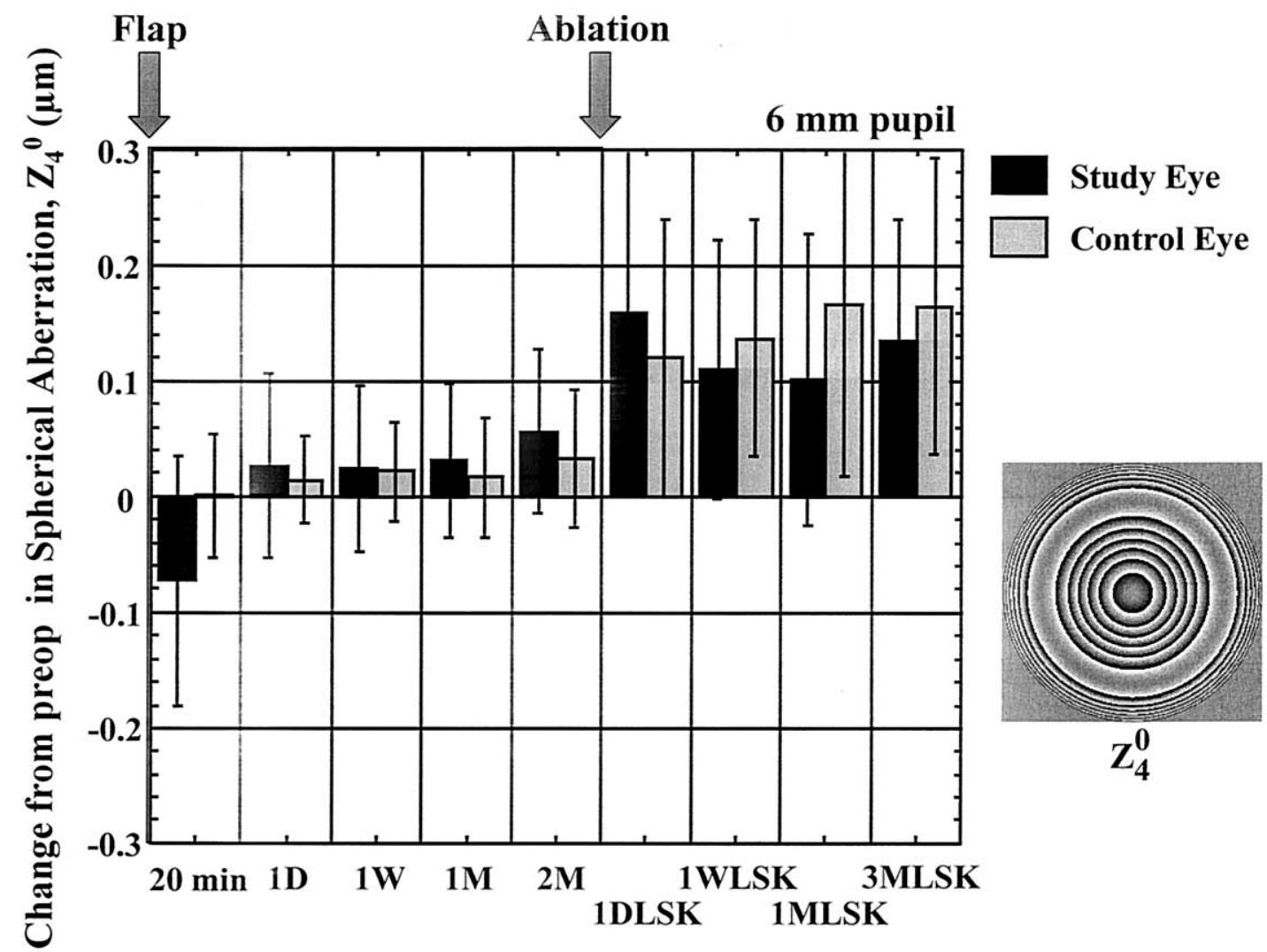

Time

FIGURE 7. Average change in spherical aberration, $Z_{4}^{\circ}$, from preoperative levels for both study eyes ( $\square$ ) and control eyes $(\square)$ for a 6-mm pupil. Positive and negative values indicate an average increase or decrease, respectively, in spherical aberration relative to preoperative values. Error bars represent \pm 1 standard deviation from the mean. Time points were 20 minutes (20 min), 1 day (1D), 1 week $(1 \mathrm{~W}), 1$ month (1M), and 2 months $(2 \mathrm{M})$ postmicrokeratome incision; and 1 day (1DLSK), 1 week (1WLSK), 1 month (1 MLSK), and 3 months ( 3 MLSK) postlaser ablation. There was no significant difference in spherical aberration between the study and control eyes 2 months $(2 \mathrm{M})$ after the microkeratome incision $(P=.36)$. Three months postablation, the spherical aberration induced in both groups was significantly different from their preoperative values $(P=.0004, P=.0003$ for study eyes and control eyes, respectively), indicating that most of the spherical aberration observed in post-laser in situ keratomileusis (post-LASIK) eyes is primarily due to the laser ablation.

minutes) in the study group when compared with the control group (Figure 5). Vertical coma decreased in the study group and was not significant when compared with the control group at 2 months after the flap cut and 3 months postablation. Pallikaris and coworkers ${ }^{21}$ noted an increase in horizontal coma after using a microkeratome that created a nasal hinge, suggesting the position of the flap hinge may influence the type(s) of aberrations induced after the incision. An analogous finding of induced vertical coma was not found for our cohort of patients after creating a flap with a superiorly located hinge. In addition to the location of the flap hinge, the type of microkeratome used to make the cut and flap thickness uniformity may impact the induced amount and orientation of the eye's higher order aberrations.

Overall, there was not a large, systematic change in the total aberration structure of the study eyes after the microkeratome incision. Despite an average approximate
$30 \%$ increase in the study eyes higher order rms wavefront error 2 months after the microkeratome incision, there were no consistent changes in any Zernike modes, with the exception of a slight, significant negative shift in one trefoil mode, $Z_{3}^{3}$. This significant change in trefoil was more pronounced immediately after the flap was created, but persisted for 2 months after the microkeratome incision in the study eyes. A possible explanation for why trefoil, and not coma, was significantly altered after the flap cut could be due to the fact that the motion of the Hansatome more resembles an arc that begins in the temporal inferior quadrant of the eye and sweeps upward to leave a superior hinge (Figure 8). This arcing cut may account for the systematic change across patients in only the Zernike trefoil term. It may be necessary to characterize the types of aberrations induced by any microkeratome used in a customized LASIK procedure to provide an optimal higher order correction. Further studies are warranted in this regard. 


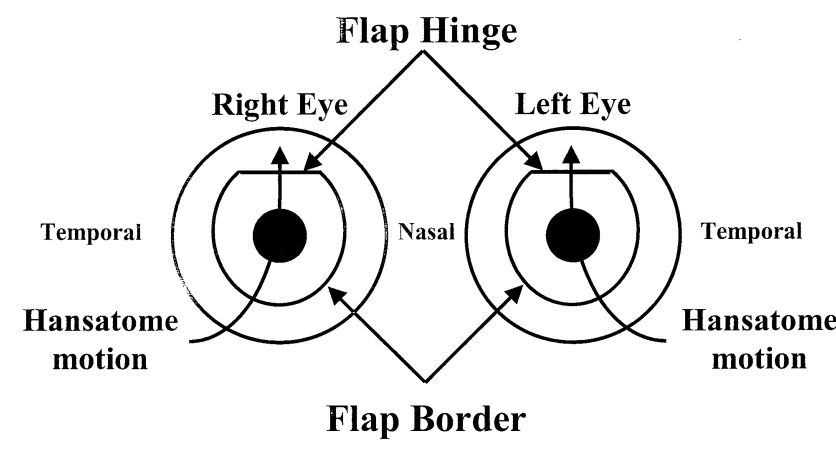

FIGURE 8. Motion of the Hansatome microkeratome on the left and right eyes. The Hansatome pivots around a temporally located post and rotates in an arc as it passes over the cornea to create a superiorly hinged flap. This arcing cut may contribute to the systematic shift in the trefoil mode, $Z_{3}^{3}$, noted in Figure 62 months after the flap cut.

An important reason for performing this study was to investigate two potential mechanisms by which spherical aberration could be induced in a LASIK procedure: the flap cut and the laser ablation. The results indicate that the majority of spherical aberration induced after LASIK is due to the laser ablation and not the microkeratome incision. The study eyes experienced an average increase in spherical aberration of $0.057 \pm 0.0706 \mu \mathrm{m} 2$ months after the microkeratome incision (Figure 7). This slight increase, however, was not significantly different when compared with the control eyes with an average increase of $0.033 \pm$ $0.0603 \mu \mathrm{m}(P=.36)$.

The sharpest increases in spherical aberration for both groups were observed after the laser ablation. The study eyes had an average attempted correction of $-2.55 \pm 1.55$ diopters sphere and experienced an average increase (relative to preoperative) of $0.136 \pm 0.1038 \mu \mathrm{m} 3$ months postablation. The control eyes, with an average attempted correction of $-2.61 \pm 1.48$ diopters sphere, had a similar average increase in spherical aberration of $0.165 \pm 0.1272$ $\mu \mathrm{m}$. Even though these average increases may appear small, the modest postablation increases in spherical aberration for our cohort of patients match typically induced amounts observed in post-LASIK patients for comparable amounts of attempted myopic correction and the same laser. Figure 9 illustrates the increase in spherical aberration 1 month post-LASIK for a given attempted correction in a separate group of 32 normal patients receiving conventional myopic treatments with the same Technolas laser (Bausch \& Lomb). ${ }^{22}$ These 32 patients had a comparable range of myopia (average of $-2.41 \pm 1.07 \mathrm{D}$ sphere) and different amounts of postoperatively induced spherical aberration, depending on their myopic correction. As predicted by the best fit line from Figure 9, an attempted correction of $-2.55 \mathrm{D}$ sphere, corresponding to the average value treated in our study eyes, would result in a postoperative increase in spherical aberration of 0.156 $\mu \mathrm{m}$. This value closely matches the $0.136 \pm 0.1038-\mu \mathrm{m}$ average increase observed in our study eyes. Similarly, the increase in spherical aberration predicted by Figure 9 for an attempted correction of $-2.61 \mathrm{D}$ sphere, the average value treated in the control eyes, would be approximately 0.160 $\mu \mathrm{m}$. This result is also comparable with the $0.165 \pm$ $0.1272 \mu \mathrm{m}$ of spherical aberration that was induced, on average, in our control eyes. If higher myopes had been enrolled in our study, a larger difference between the amount of spherical aberration induced 2 months postflap cut and 3 months postablation would most likely have been observed.

The slight hyperopic shift that occurred after the flap cut and the prevalence of the laser ablation in inducing spherical aberration are consistent with findings from previous investigators. Roberts and other authors have noted an increased flattening and hyperopic shifts in studies on PTK and myopic PRK and LASIK. ${ }^{13-17}$ This effect can be attributed to a severing and retraction of the collagen lamellae. Corneal topography reveals this thickening and steepening of the midperiphery with the "red ring," which is associated with positive spherical aberration. The central flattening associated with midperipheral steepening occurs because curvature must be conserved if the surface is to be smooth and continuous when the cord length of the cornea does not change. ${ }^{23}$ This current study helps clarify this biomechanical mechanism by noting that, upon manifest refraction, only a mild average hyperopic shift of 0.28 diopters occurred in the study eyes 2 months after the flap cut. We also found that the laser ablation dictates the marked central flattening and midperipheral steepening caused by LASIK and that the microkeratome incision plays only a minor role. The marked increase in positive spherical aberration noted by Oshika and coworkers ${ }^{5}$ after PRK supports our conclusion that the increase in spherical aberration in LASIK and PRK/ LASEK is caused primarily by the laser ablation.

There are several mechanisms that could account for the increases in spherical aberration and the higher order aberrations that are typically observed in conventional post-LASIK eyes. This study has demonstrated that, in addition to a slight hyperopic shift, there is a slight, nonsystematic increase in the eye's higher order aberrations (with the exception of trefoil) after the microkeratome cut. These changes, however, can not entirely account for the total amount of postoperative increase in higher order aberrations usually encountered after LASIK. The results also indicate that the laser ablation induces the majority of postoperative spherical aberration. Much work must still be done to investigate the relative impact of several other factors on the final aberration structure of the postoperative LASIK eye. Some of these factors include determining the efficiency of laser pulses striking the cornea perpendicularly vs obliquely, ${ }^{24}$ properly registering corneal topographic, wavefront and laser ablation axes, determining how quickly and accurately eye position and 


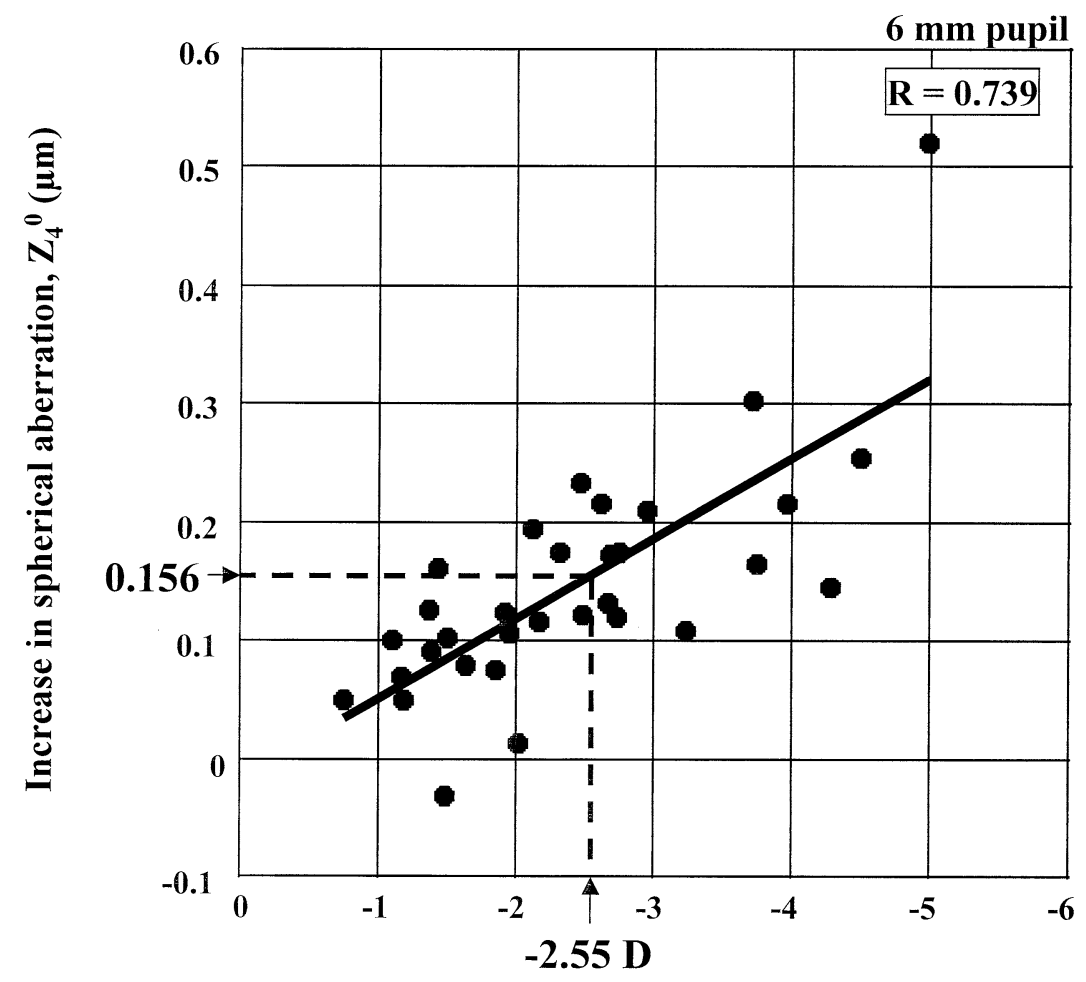

\section{Preoperative defocus (D)}

FIGURE 9. Amount of increase in spherical aberration after conventional myopic laser in situ keratomileusis (LASIK) surgery in a separate group of 32 eyes as a function of preoperative levels of defocus (6-mm pupil). There is a strong positive correlation ( $\mathrm{r}$ $=0.739$ ) between the amount of spherical aberration induced after LASIK surgery and the degree of intended correction. The small average increase in spherical aberration observed 3 months postablation in the study eyes of $0.136 \pm 0.1038 \mu \mathrm{m}$ (average attempted correction of -2.55 diopters) agrees nicely with the value of $0.156 \mu \mathrm{m}$ predicted by this plot.

movements need to be tracked during the procedure, ${ }^{25-27}$ being able to model and predict the postoperative healing responses of the cornea, and determining limits imposed on customized postoperative outcomes due to surgeon and environmental variability. Only by examining the separate effects of each of these factors on the final refractive surgery outcomes will it be possible to understand their relative contributions and potential interactions to optimize a customized correction of the eye's optics.

\section{REFERENCES}

1. Mrochen M, Kaemmerer M, Seiler T. Wavefront-guided laser in situ keratomileusis: early results in three eyes. J Refract Surg 2000;16:116-121.

2. Seiler T, Mrochen M, Kaemmerer M. Operative correction of ocular aberrations to improve visual acuity. J Refract Surg 2000;16:S619-S622.

3. Mrochen M, Kaemmerer M, Seiler T. Clinical results of wavefront-guided laser in situ keratomileusis 3 months after surgery. J Cataract Refract Surg 2001;27:201-207.

4. Panagopoulou SI, Pallikaris IG. Wavefront customized ablations with the WASCA Asclepion workstation. J Refract Surg 2001;17:S608-S612.
5. Oshika T, Klyce SD, Applegate RA, Howland HC, El Danasoury MA. Comparison of corneal wavefront aberrations after photorefractive keratectomy and laser in situ keratomileusis. Am J Ophthalmol 1999;127:1-7.

6. Thibos LN, Hong X. Clinical applications of the ShackHartmann Aberrometer. Optom Vis Sci 1999;76:817-825.

7. Williams DR, Yoon GY, Porter J, Guirao A, Hofer H, Cox I. Visual benefit of correcting higher order aberrations of the eye. J Refract Surg 2000;16:S554-S559.

8. Mrochen M, Kaemmerer M, Mierdel P, Seiler T. Increased higher-order optical aberrations after laser refractive surgery: a problem of subclinical decentration. J Cataract Refract Surg 2001;27:362-369.

9. Endl MJ, Martinez CE, Klyce SD, et al. Effect of larger ablation zone and transition zone on corneal optical aberrations after photorefractive keratectomy. Arch Ophthalmol 2001;119:1159-1164.

10. Moreno-Barriuso E, Lloves JM, Marcos S, Navarro R, Llorente L, Barbero S. Ocular aberrations before and after myopic corneal refractive surgery: LASIK-induced changes measured with laser ray tracing. Invest Ophthalmol Vis Sci 2001;42:1396-1403.

11. Howland HC, Rand RH, Lubkin SR. A thin-shell model of the cornea and its application to corneal surgery. Refract Corneal Surg 1992;8:183-186.

12. Roberts C. The cornea is not a piece of plastic. J Refract Surg 2000;16:407-413. 
13. Roberts C, Dupps WJ Jr. Corneal biomechanics and their role in corneal ablative procedures. In: MacRae S, Krueger R, Applegate R, eds. Customized corneal ablation. Thorofare, NJ: Slack Inc., 2001:109-131.

14. Dupps WJ Jr, Roberts C. Effect of acute biomechanical changes on corneal curvature after photokeratectomy. J Refract Surg 2001;17:658-669.

15. Sher NA, Bowers RA, Zabel RW, et al. Clinical use of the 193-nm excimer laser in the treatment of corneal scars. Arch Ophthalmol 1991;109:491-498.

16. Gartry D, Kerr Muir M, Marshall J. Excimer laser treatment of corneal surface pathology: a laboratory and clinical study. Br J Ophthalmol 1991;75:258-269.

17. Rogers C, Cohen P, Lawless M. Phototherapeutic keratectomy for Reis Bucklers' corneal dystrophy. Aust N Z J Ophthalmol 1993;21:247-250.

18. Thibos LN, Applegate RA, Schwiegerling JT, Webb R, VSIA Standards Taskforce Members. Standards for reporting the optical aberrations of eyes. OSA trends in optics and photonics (Vol. 35). In Lakshminarayanan V, ed. Vision science and its applications. Washington, D.C.: Optical Society of America, 2000:232-244.

19. Porter J, Guirao A, Cox IG, Williams DR. Monochromatic aberrations of the human eye in a large population. J Opt Soc Am A 2001;18:1793-1803.

20. Thibos LN, Hong X, Bradley A, Cheng X. Statistical variation of aberration structure and image quality in a normal population of healthy eyes. J Opt Soc Am A 2002;19:2329-2348.

21. Pallikaris IG, Kymionis GD, Panagopoulou SI, Siganos CS, Theodorakis MA, Pallikaris AI. Induced optical aberrations following formation of a laser in situ keratomileusis flap. J Cataract Refract Surg 2002;28:1737-1741.

22. Cox IG. Clinical results of customized ablations: Bausch $\&$ Lomb. Proceedings of the $3^{\text {rd }}$ International Congress of Wavefront Sensing and Aberration-Free Refractive Correction 2002 (Interlaken, Switzerland).

23. MacRae S. Excimer ablation design and elliptical transition zones. J Cataract Refract Surg 1999;25:1191-1197.

24. Mrochen M, Seiler T. Influence of corneal curvature on calculation of ablation patterns used in photorefractive laser surgery. J Refract Surg 2001;17:S584-S587.

25. Taylor NM, Eikelboom RH, van Sarloos PP, Reid PG. Determining the accuracy of an eye tracking system for laser refractive surgery. J Refract Surg 2000;16:S643-S646.

26. Yoon GY, Kwon C, MacRae S, Porter J, Cox I, Williams DR. Effect of corneal decentration on the outcome of laser refractive surgery procedure. Proceedings of $3^{\text {rd }}$ International Congress of Wavefront Sensing and Aberration-Free Refractive Correction 2002 (Interlaken, Switzerland).

27. Gobbi PG, Carones F, Brancato R, et al. Automatic eye tracker for excimer laser photorefractive keratectomy. J Refract Surg 1995;11(3, Suppl):S337-S342. 